\title{
Etude de Perception de l'employabilité chez les étudiants issus des établissements à accès régulé dans le secteur public au Maroc : vers un style pédagogique favorisant l'autonomie
}

\author{
Melhaoui Issam, Docteur en sciences de gestion \\ Balhadj Said, Professeur de l'enseignement supérieur \\ Ecole Nationale de Commerce et de Gestion de Tanger \\ Université Abdelmalek Essaadi, Morocco
}

Doi:10.19044/esj.2021.v17n32p141

Submitted: 21 August 2021

Accepted: 21 September 2021

Published: 30 September 2021
Copyright 2021 Author(s)

Under Creative Commons BY-NC-ND

4.0 OPEN ACCESS

Cite As:

Issam M. \& Said B. (2021). Etude de Perception de l'employabilité chez les étudiants issus des établissements à accès régulé dans le secteur public au Maroc : vers un style pédagogique favorisant l'autonomie. European Scientific Journal, ESJ, 17 (32), 141.

https://doi.org/10.19044/esj.2021.v17n32p141

\section{Résumé}

L'intérêt porté à la pédagogie universitaire et à l'employabilité des jeunes diplômés, réside dans l'ambition d'améliorer la qualité du système éducatif. Et ce, par une mise en question rigoureuse sur le style pédagogique autonomisant du professeur, et son incidence sur les déterminants individuels de l'employabilité. Le présent travail vise à étudier l'effet potentiel du style pédagogique autonomisant sur la perception de l'employabilité des étudiants. Afin de confronter les aboutissements théoriques à la réalité marocaine, nous avons mené une étude qualitative auprès des étudiants en fin de parcours issus des écoles publiques à accès régulé du secteur Commerce, Gestion, et Sciences de l'ingénieur. Les résultats montrent qu'effectivement les jeunes étudiants sont plus susceptible de développer des caractéristiques individuelles et des compétences d'employabilité par la perception d'un style pédagogique autonomisant.

Mots-clés: Employabilité, pédagogie, autonomie, motivation, autodétermination, auto efficacité 


\title{
Study on the Perception of Employability Among Students from Regulated Access Institutions in the Public Sector in Morocco: Towards a Pedagogical Style that Promotes Autonomy
}

\author{
Melhaoui Issam, Docteur en sciences de gestion \\ Balhadj Said, Professeur de l'enseignement supérieur \\ Ecole Nationale de Commerce et de Gestion de Tanger \\ Université Abdelmalek Essaadi, Morocco
}

\begin{abstract}
The interest in university pedagogy and the undergraduate's employability lies in the ambition to improve the quality of the educational system. This is done through a rigorous questioning of the autonomy supportive teaching style and its impact on the individual determinants of employability. The present work aims to investigate the potential effect of autonomy supportive teaching style on students' perceived employability. In order to confront the theoretical findings with the Moroccan reality, we conducted a qualitative study with undergraduates from public schools with regulated access in the business, management, and engineering sectors. The results show that young students are indeed more likely to develop individual characteristics and employability skills through the perception of autonomy supportive teaching style.
\end{abstract}

Keywords: Employability, pedagogy, autonomy, motivation, selfdetermination, self-efficacy

\section{Introduction}

De nos jours, le paysage des études supérieures a changé, sous l'influence de la mondialisation et des changements socio-économiques, l'employabilité est devenue l'objectif principal des établissements de formation. Dans un contexte où le rôle important de l'université ne s'explique plus, ou parait paradoxal avec l'employabilité perçue (taux de chômage important, taux d'encadrement pédagogique en chute, le manque de rapports sur l'état de l'employabilité des étudiants,etc.). Il serait donc important d'étudier et d'analyser l'employabilité dans le contexte marocain d'un point de vue pédagogique.

L'employabilité est un concept multidimensionnel qui s'est développé assez largement depuis une trentaine d'années, toutefois, il n'existe pas encore un consensus théorique sur la définition de ce concept. C'est pourquoi, dans 
le cadre de notre recherche, nous focalisons sur l'étudiant considéré comme l'unité de base de notre analyse. L'employabilité est donc appréhendée dans ce travail comme une responsabilité individuelle de l'étudiant détaché de tout lien avec les caractéristiques du marché de travail (sans pour autant omettre l'importance de cette dimension dans l'analyse de l'employabilité). De nombreuses recherches ont tentés d'étudier le développement de l'employabilité dans le contexte universitaire du point de vue individuelle de l'étudiant (Harvey, 2001, Yorke et Knight 2004, Dacree Lorraine et Sewell, 2007).

Conformément à la revue de littérature, la pédagogie traditionnelle connaît des limites quant à l'employabilité des étudiants, selon (Lemelin, 2015, p :2), "le fait que les cours magistraux soient le modèle dominant soulève des questions quant à l'employabilité. En ne misant essentiellement que sur le savoir, il laisse de côté le savoir-faire et le savoir-être qui constituent des éléments importants de l'employabilité ». Dans ce cadre, les nouvelles pratiques pédagogiques -modèles pédagogiques de la demande centrée sur l'étudiant- renvoient à une nouvelle psychopédagogie des adultes basée sur un style émancipateur.

Conformément à la littérature, il parait qu'un style pédagogique autonomisant s'associe corrélativement à une motivation intrinsèque et un fort degré d'auto efficacité, alors qu'un style contrôlant conduit à des motivations extrinsèques et un faible degré d'auto efficacité chez les étudiants (Leroy, 2009; Leroy et al., 2013; Reeve, 2006; Reeve et al., 2004b; Sarrazin et al., 2011). Nous constatons que le concept du « style pédagogique autonomisant », a été relié positivement à la motivation intrinsèque (Reeve, Nix, et Hamm, 2003), au développement des croyances d'efficacité des étudiants (Nadia Leroy et al 2006,2013), à l'engagement des étudiants (Reeve, 2006,2012), au bien-être (Black et Deci, 2000). Toutefois, l'employabilité n'a pas fait l'objet de liaison avec ce concept.

Afin de répondre à l'objectif de ce travail, nous allons présenter dans un premier temps les concepts et le cadre théorique relatif au concept de style pédagogogique autonomisant et l'employabilité « conditionnée par le système éducatif » puis nous allons tenter de montrer dans le cadre d'une étude qualitative l'effet du style pédagogique autonomisant sur la perception de l'employabilité des étudiants diplômés.

\section{Le style pédagogique autonomisant et son influence sur la perception de l'employabilité des diplômés : cadre théorique}

\subsection{Fondement théorique de la pédagogie universitaire}

La pédagogie selon le dictionnaire de la pédagogie c'est « la science de l'éducation tant physique, qu'intellectuelle ou morale », nous retenons également la définition de Margueritte Altet (2006) « le champ de la 
transformation de l'information en savoir par la médiation de l'enseignant, par la communication, par l'action interactive dans une situation éducative donnée $»$.

Le champ théorique qui encadre la pédagogie est très vaste et diversifié, c'est pourquoi pour parler de pédagogie ou apprentissage, les chercheurs se sont mis d'accord sur les paradigmes sur lesquels ressortent toutes les méthodes pédagogiques et d'apprentissages, à savoir «le positivisme », «le behaviorisme », «le constructivisme», «le sociocognitivisme $\gg$ etc.

La théorie sociocognitive fondé par Albert Bandura et particulièrement pertinente pour le cas de notre recherche, car elle offre une vision plus riche de la réalité ne se limitant pas à la relation binaire ou unidirectionnel dans l'acte d'apprendre ou d'enseigner. En effet elle remet en cause le fonctionnement binaire ou unidirectionnel de l'opération apprentissageenseignement, comme l'explique Bandura (1999) « les personnes ne réagissent pas simplement aux influences de leur environnement immédiat comme des girouettes, de même qu'elles ne sont pas mécaniquement gouvernées par leur passé... Les théories qui cherchent à expliquer le comportement humain uniquement comme le produit des influences extérieures ou des vestiges d'anciens stimuli présentent une image tronquée de la nature humaine. La capacité à agir de façon intentionnelle et volontaire est enracinée dans l'activité symbolique ».

Albert Bandura propose donc un modèle triadique qui se base sur la relation « personnes » « croyances » « environnement » comme présenté dans le modèle ci-dessous.

De ce fait notre position sociocognitive s'explique, sur l'aspect riche et complémentaire de cette théorie, ainsi, sa relation directe avec l'objet de recherche considérant l'étudiant comme l'unité de base de l'analyse et considérant les étudiants comme des agents actifs, c'est-à-dire des agents qui ont la capacité d'anticiper et réguler leurs comportements.

De ce paradigme ressortent plusieurs théories qui ont influencé la manière d'apprendre et d'enseigner vers une psychopédagogie qui se concentre sur le développement identitaire de l'étudiant. La théorie de l'apprentissage autorégulé qui s'inscrit dans l'approche sociocognitive ( Zimmerman 2002, Cofresnoy (2010) explicite clairement l'importance de la motivation dans le processus d'apprentissage des adultes. En effet, la motivation permet aux étudiants de persévérer et résister dans l'apprentissage, comme expliqué par Cofresnoy (2010) aujourd'hui, les aspects cognitifs ne sont plus suffisants. Car, au-delà des pédagogies actives, il faut surtout que les activités d'enseignement transmettent un sentiment d'autonomie et crée un intérêt chez les étudiants, c'est pourquoi la théorie de l'apprentissage autorégulé permet une explication du comportement d'apprentissage en 
prenant en considération les facteurs cognitifs, métacognitifs et motivationnels.

D'un autre côté, Selon P.Carré (2015), la formation (pédagogie) ne fait pas forcément l'acte d'apprendre, mais plutôt crée les conditions favorisant l'apprentissage, car, le sens produit dans la formation par le professeur selon carré est toujours médié par la compréhension et la disposition (c'est-à-dire refus ou consentement par rapport à ce qui a été compris), C'est pourquoi il est crucial de s'intéresser à la motivation et comportements d'apprentissage des étudiants.

\subsection{Le style pédagogique autonomisant :}

Le champ théorique qui encadre la pédagogie universitaire est assez large et diversifié relevant de la philosophie, psychologie, sciences cognitives etc. nous nous sommes concentrés dans le cas de ce travail à l'étude du style pédagogique et son lien avec les processus motivationnels de l'étudiant. Il semble selon plusieurs recherches que le concept du «style pédagogique autonomisant », développé par Johnmarshall Reeve, a été relié positivement à la motivation intrinsèque (Reeve, Nix, \& Hamm, 2003), au développement des croyances d'efficacité des étudiants (Nadia Leroy \& al 2006,2013), à l'engagement des étudiants (Reeve, 2006,2012), au bien-être (Black \& Deci, 2000). Toutefois, l'employabilité n'a pas fait l'objet de liaison avec ce concept.

Selon Philippe Sarazzin, le concept de style d'enseignement ou style pédagogique a vu le jour depuis des décennies pour étudier le comportement et pratique de l'enseignant. Pour (Legendre, 1993, P:986), le style d'enseignement est la "configuration de comportements et d'attitudes (faits et gestes, intérêts, communications, caractère) qui caractérise un enseignant en regard des composantes et des diverses relations d'une situation pédagogique". Pour (Creemers, 1974), le style d'enseignement est "l'ensemble des activités méthodologiques que l'enseignant met en œuvre pour déclencher et soutenir l'apprentissage." (Creemers, 1974, p:124) cité également dans (Karsenti, 1993, P:54) Selon (Altet, 2002) Le style d'enseignement « Peut se définir à partir d'une observation des activités (actions/comportements) de l'enseignant à l'intérieur d'un contexte précis. »(Altet, 2002).

Les travaux de Sarrazin $(2006,2011,2013)$; Reeve $(2004,2008,2012)$; Deci\&Ryan (2006), se sont intéressés, particulièrement, à étudier les influences du style pédagogique autonomisant sur la motivation et les performances académiques. Ces derniers font ressortir l'importance d'encourager l'instauration d'un climat motivationnel autonomisant en classe. Comme le confirme Reeve (2006) : "Les élèves des classes enseignées par des enseignants qui soutiennent l'autonomie, par rapport aux élèves des classes enseignés par des enseignants contrôlants, font l'expérience d'une gamme 
impressionnante et significative de résultats éducatifs positifs.».(Reeve, 2006, P:228) Selon (Sarrazin et al, 2011). Le style pédagogique autonomisant c'est : «la volonté délibérée du superviseur de prendre en compte le point de vue du supervisé et de valoriser toutes les opportunités de son épanouissement personnel. À l'inverse, un style sera qualifié de « contraignant» quand la volonté délibérée du superviseur est de contraindre le supervisé à penser ou à se comporter d'une certaine façon. » (Sarrazin et al., 2011, P :306).

Le style pédagogique autonomisant se définit selon (Leroy et al., 2013, p :73) comme : « comportements qu'une personne adopte envers une autre personne en vue de promouvoir les intentions volitives ainsi que les besoins psychologiques de cette dernière ». Le style pédagogique contrôlant pour eux, c'est : « un ensemble de pratiques accentuant le poids des pressions et ayant pour but d'inciter les élèves à agir d'une façon spécifique » (Leroy et al., Idem).

L'enseignant qui soutient l'autonomie de ses élèves/étudiants tend généralement à introduire des activités qui soutiennent les besoins psychologiques à savoir, le besoin d'autonomie qui caractère le besoin de se sentir à l'origine de ces actions, Le besoin de compétence expliqué par(Sarrazin et al., 2011, P:160). comme « le désir d'interagir efficacement avec l'environnement, finalement, la proximité sociale qui renvoie au besoin de se sentir connecté affectivement ou d'appartenir à un groupe (Baumeister \& Leary, 1995)», cité par (Leroy et al., 2013, P:3). Conformément à cette théorie, l'étudiant parvient à développer des caractéristiques individuelles par la perception et la satisfaction des besoins psychologiques.

\subsection{Le rôle de la motivation dans la régulation du comportement}

Les recherches sur la motivation qui s'inscrivent dans l'approche sociocognitive sont nombreuses, dans cette perspective, la théorie des buts d'accomplissement (Nicholls, 1984, 1989 ; Elliot, 1997), la théorie de l'autodétermination (Deci et Ryan 1985, 1991, 2000) ou encore le modèle hiérarchique de Vallerand (Vallerand, 1997, 2001), la théorie de l'autoefficacité de Bandura (1986, 2003, 2007), la dynamique motivationnelle de Viau $(2000,2003,2007)$ ont fournis des éléments de réponses robustes et solides.Nous proposons dans ce sens, la définition de la motivation de vallerand et al (1993) cité par Thierry et Al (2015) : «la motivation est le construit hypothétique utilisé afin de décrire les forces internes et/ou externes produisant le déclenchement, la direction et la persistance du comportement (Vallerand et Thill, 1993, p.18) ».

Deux théories importantes sont retenues dans notre travail, en raison de leur pertinence théorique, et le grand intérêt porté à ces éléments dans les modèles de l'employabilité. Il s'agit de la théorie de l'autodétermination (Deci\&Ryan, 2002) et de l'auto-efficacité (Bandura, 2003). 
La Théorie de l'autodétermination (Deci \& Ryan, 2002) est fondamentale pour rendre compte des comportements des individus (étudiants), en effet, selon ces auteurs, plusieurs types de motivation peuvent être repérés et classés en fonction de leur degré d'autodétermination. L'intérêt de cette théorie est que l'on peut réguler le comportement des individus, car, à travers la régulation (régulation introjectée, externe.) qui se manifeste par le renforcement ou l'entrave de trois besoins psychologique (le sentiment d'autonomie, le sentiment de compétence, la proximité sociale. L'étudiant change vers un comportement autodéterminé. La théorie de l'auto-efficacité de Bandura (1986) montre que Les individus sont à la fois «producteurs et produit de leur société » A.Bandura (1986), en effet, par son modèle causale triadique, illustre le fait que l'individu est socialement constitué mais qu'il est aussi en partie à l'origine de ce qu'il fait et de ce qui lui arrive, car, il exerce une influence sur lui-même Bandura (1986). Ceci renvoie à l'idée que l'individu il est capable d'anticiper les événements et en général à influencer sur ce qu'il fait (G.joet, 2010), plus précisément, il convient de dire, que le fonctionnement humain n'est pas seulement régulé par des stimuli extérieurs, en effet, l'action peut être initié par l'individu lui-même, et auto-observé et régulé de façon a influencé ces perceptions d'efficacité, d'où l'importance du concept initié par bandura le sentiment d'efficacité personnel, ou l'autoefficacité. Selon Bandura (2003) : «il est absolument nécessaire que les stratégies de formation d'aujourd'hui tiennent compte d'un environnement professionnel devenu très instable, en guidant les apprenants vers l'autonomie de formation et la prise en charge de leur propre besoin et de leur développement personnel, tout en les persuadant de leur capacité d'apprentissage » ces éléments permettront de développer leurs croyances d'efficacité et par conséquent leur motivation.

\subsection{L'employabilité des jeunes diplômés : les déterminants au niveau des caractéristiques individuelles}

\subsubsection{L'employabilité : un concept multidimensionnel}

Selon hofaidhllaoui (2014) l'employabilité, peut être défini à plusieurs niveaux : au niveau de la société et de l'économie (Ledrut, 1966 ; Finn, 2000 ; bollérot, 2002 ; De Grip et al., 2004), au niveau de l'organisation (De Vries et al., 2001 ; Saint-Germes, 2006) et au niveau de l'individu (Kluytmans et ott, 1999 ; Finot, 2000 ; Dany, 1997; Van buren iii, 2003 ; Van Dam, 2004 ; Fugate et al., 2004).

L'employabilité selon Hofaidhllaoui (2011): «est présente dans l'entreprise, sur le marché de l'emploi, dans les stratégies individuelles, notamment celles que développent les jeunes diplômés. Dans chacun de ces univers, elle recouvre des réalités multiples », nous pouvons dire alors que 
cette notion d'employabilité a acquis plusieurs significations et plusieurs définitions avec le temps.

Compte tenu de son caractère multidimensionnel, l'employabilité reste un terme flou qui s'est développé assez largement avec le temps mais sans aboutir à une définition universelle de l'employabilité. Toutefois, les chercheurs semblent s'accordé sur trois éléments fondamentaux. La définition de Hillage et Pollard (1998) nous semblent approprié pour illustrer ces trois éléments :

« 1- la capacité à obtenir un emploi initial (initial employment), conditionnée entre autres par le système éducatif, 2- La capacité à conserver son emploi et à mener les transitions entre les emplois et les rôles dans une même organisation, 3- La capacité à trouver un autre emploi si nécessaire, entendue comme la capacité et la volonté des individus dans le management des transitions d'emploi entre les organisations et en leur sein » cité par Eve Saint Germes (2004).

L'employabilité conditionné par le système éducatif selon Lemelin (2015) : « dépend des connaissances, des qualifications et des comportements qu'on a, de la façon dont on s'en sert et dont on les présente à l'employeur. ». le système de formation constitue une phase importante pour les étudiants pour se doter des compétences et des qualités individuelles indispensable à la carrière des futurs diplômés, selon Harvey (2001) les établissements de formation permettent du moins de développer une pré-employabilité ou ce qu'il est nommé par l'auteur «employability devlopment opportunities » susceptible de traduire éventuellement les capacités et les compétences acquises en employabilité future réelle. Selon maurice lemelin par la formation académique les étudiants développent ou du moins améliorent un savoir, savoir-faire et savoir-être qui constituent des éléments importants à l'employabilité des jeunes et ce par une pédagogie active qui conditionne une employabilité réelle.

\subsubsection{Les déterminants au niveau des caractéristiques individuelles.}

Des chercheurs anglo-saxons tels que (Hillage et pollard 1996, Harvey 1998, Fugate et Al, 2004, Yorke\& knight 2002,2006, D. Pool et sewell 2007) ont bâtis une revue de littérature robuste sur les déterminants individuels de l'employabilité des jeunes diplômés. Trois grandes catégories de déterminants individuels sont à distinguer : les attitudes, les compétences, la personnalité. Selon fugate et $\mathrm{Al}$ (2004, 2008), un ensemble de caractéristiques psychologiques centrés sur la personne sont capables d'influencer sur l'employabilité, considérée dans cette approche comme un construit psychosocial : 
"Employability is a psycho- social construct that embodies individual characteristics that foster adaptive cognition, behavior, and affect, and enhance the individual-work interface." Fugate (2004, p:2)

Nous pouvons retenir de cette définition que l'employabilité demande une personnalité proactive, capable de faire face aux changement de l'environnement comme le souligne E.Mercier(2011):

«L'individu employable est une personne proactive face aux changements, capable de détecter les bonnes opportunités sur le marché, d'obtenir de l'information utile via son réseau professionnel, et qui sait donner du sens à ses choix grâce à une forte identité. » Il en découle que la clé pour rester activement employable repose selon Fugate et Al (2004) sur 3 dimensions fondamentales : l'adaptation personnelle (personal adaptability), l'identité de carrière (career identity), capital humain et social (social and humain capital).

Van Der Heijden et Van Der Heijde (2004), de leurs parts, estiment que les compétences ont un pouvoir explicatif, quant à l'employabilité. L'employabilité basée sur l'approche par compétences se définie comme : « l'accomplissement, l'acquisition ou la création permanents de travail grâce à une utilisation optimale des compétences. » Frimousse Ali et al (2013)

Selon frimousse et al (2013) dans cette perspective l'employabilité représente une combinaison des compétences individuelles spécifiques (liée à l'expertise professionnelle) et générales (compétences sociales et compétences liées à la flexibilité) qui peuvent se résumer 5 éléments essentiels : l'expertise professionnelle, L'anticipation et l'optimisation, La flexibilité personnelle, L'esprit collectif, Balance

Finalement, La vision attitudinale de l'employabilité selon hofaidhllaoui (2011) met en exergue l'adaptabilité de la personne. Cette dernière peut se décliner dans trois directions : organisationnelle, fonctionnelle, sectorielle, que trois études ( MHofaidhllaoui, 2011).

Nous constatons, le caractère complexe de l'employabilité qui a conduit à garder l'aspect décortiqué de l'ensemble des déterminants, par contre, au niveau de la revue de littérature anglo-saxonne, les études britanniques surtout, montrent l'intérêt de combiner ces différents déterminants. En fait, dans le cadre de développement de l'employabilité des diplômés, Yorke and Knight (2004) ont publiés un rapport nommé < skills plus $>$ dans lequel, un modèle intitulé USEM explicite clairement, L'idée que l'employabilité n'en découle pas seulement des compétences et connaissances acquises dans le système éducatif, mais de plusieurs facteurs sociaux et psychologique liés entre eux. Ceci permet au sens de Sumanasiri et al (2015) de rendre compte au mieux la complexité humaine.

Selon (yorke \& knight, 2004) les facteurs déterminants de l'employabilité des diplômés s'articulent sur les compétences des diplômés 
(skills), connaissances académiques acquises (subject understanding), et les attributs personnels (personal attributes). C'est ainsi que la definition de l'employabilité des diplômés peut se proposer comme suit: "Employability is having a set of skills, knowledge, understanding and personal attributes that make a person more likely to choose and secure occupations in which they can be satisfied and successful" (yorke et knight, 2006)

Dans la même ligne d'idées des auteurs précédents, D.Pool et Al (2007) propose un autre modèle nommé « CareerEDGE » Ce modèle identifie cinq éléments essentiels : L'expérience (travail, vie), performance académique (Subject Understunding), compétences génériques, intelligence émotionnelle, apprentissage et carrière (career developpement learning) qui sont considérés comme les clés de l'employabilité des jeunes diplômés.

\subsection{Proposition d'un modèle Théorique : l'effet d'un style pédagogique autonomisant sur l'employabilité individuelle des diplômés}

Nos aboutissements théoriques se concrétisent sous forme d'un modèle théorique qui fait le lien entre la perception du style pédagogique instauré par le professeur en classe et la perception l'employabilité. En effet, nous constatons de la revue de littérature, le rôle important du professeur dans le développement identitaire des diplômés, et ces répercussions sur la perception de l'employabilité des diplômés.

Certes, le professeur peut déclencher une grande confiance et persévérance chez les étudiants, selon la théorie de l'autodétermination, nous avons expliqué que le style pédagogique autonomisant autorapporté par le professeur peut influencer sur les perceptions et le comportement de l'étudiant, d'un comportement contraint émanant d'une force extérieure (motivation non autodéterminée) à un comportement autonome, émanant d'une force intérieure (motivation autodeterminée), ainsi, la théorie de l'autoefficacité stipule que ce dernier peut être considérer comme une source importante du sentiment d'efficacité personnelle, particulièrement, par la persuasion verbale, Selon Bandura (2003, p. 154) «La persuasion sociale permet elle aussi de renforcer les croyances des personnes qu'elles possèdent les capacités d'obtenir ce qu'elles souhaitent. Il est plus facile à quelqu'un de maintenir un sentiment d'efficacité, particulièrement quand il est confronté à des difficultés, si d'autres individus significatifs lui expriment leur confiance en ses capacités que s'ils manifestent des doutes "

Par ailleurs, dans une perspective de recherche de déterminants individuels de l'employabilité, L'employabilité en contexte anglosaxon surtout, est souvent appréhendé responsabilité de l'individu détaché de tout lien avec la vision du marché de travail et les caractéristiques du marché de l'emploi. 
Dans ce cadre, au niveau conceptuel selon (J.Othmane et ,2011, M.Hofaidhllaoui et al,2014...) Trois facteurs majeurs de l'employabilité individuelle ont été mises en évidence par une littérature abondante : les attitudes, les traits de personnalité et les compétences, nous proposons au niveau de notre modèle une version combinée des dimensions citées ci-dessus et qui s'inspire directement des travaux de ( M.Yorke et al, 2004, D. Lorraine, 2017...).

A ce niveau, l'employabilité des diplômés selon D.Lorraine et $\mathrm{AL}(2012)$ peut se définir : Employability is having a set of skills, knowledge, understanding and personal attributes that make a person more likely to choose and secure occupations in which they can be satisfied and successful».

Notre Modèle présenté ci-dessus est construit en s'inspirant des modèles suivants : Modèle de motivation en contexte académique de N. Leroy (2013), Modèle portant sur les déterminants d'employabilité J.Othmane (2011), Modèle de D. Lorraine et Al (2007) portant sur l'employabilité des diplômés.

Figure 1 : Modèle théorique de développement de l'employabilité individuelle en contexte académique

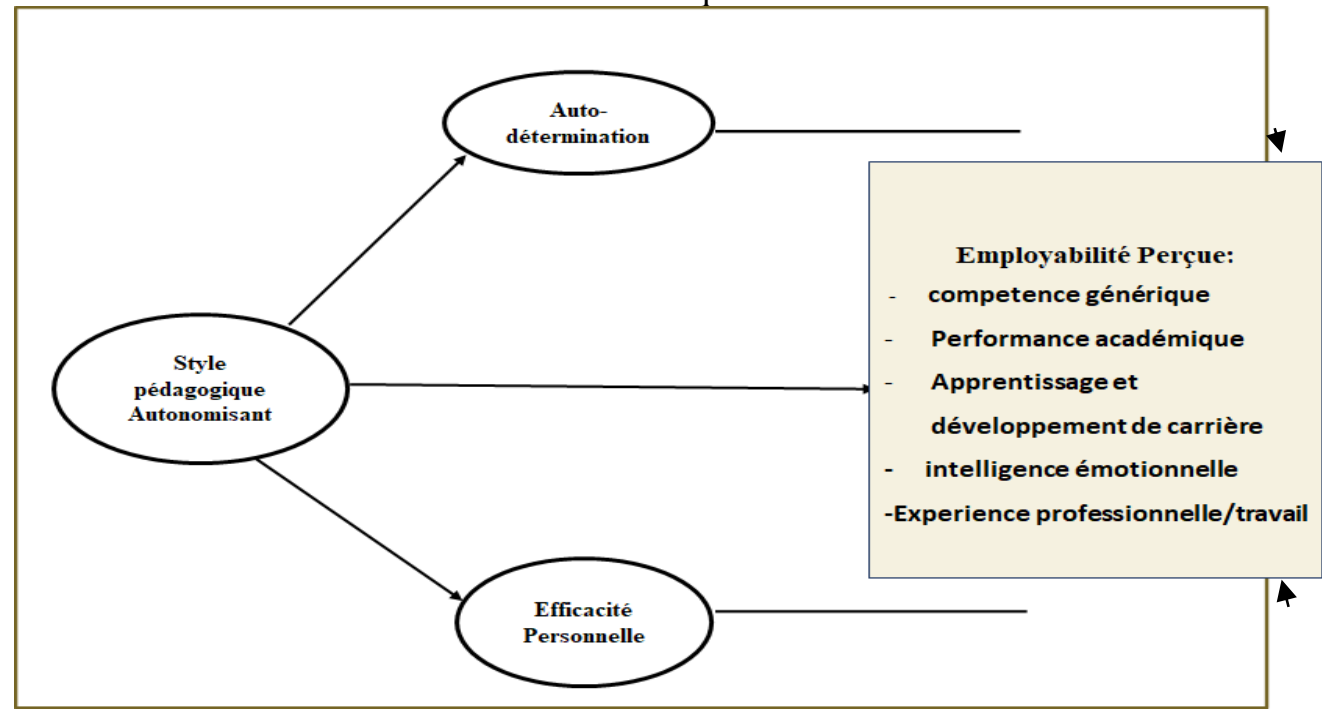

Sur la base des aboutissements théoriques, nous formulons les hypothèses suivantes :

Hypothèse 1,2 : Un style d'enseignement qui soutient l'autonomie des élèves est relié positivement d'une part aux croyances d'auto-efficacité et à l'autodétermination des étudiants/diplômés. (N. Leroy et Al, 2010,2013).

Hypothèse 3 : l'autodétermination (sentiment d'autonomie) influence positivement sur_l'employabilité des jeunes Diplômés ou pré-employabilité des étudiants (J. Othmane, Khlifi 2011,2014). 
Hypothèse 4 : Le sentiment d'efficacité personnelle influence positivement l'employabilité des jeunes diplômés (Yorke\&Knight 2002, Fugate et Al 2004, D.Lorraine 2007, Othmane 2011).

Hypothèse 5 : l'enseignement soutenant l'autonomie influence positivement L'employabilité les jeunes diplômés (J.Othmane, 2011; Aouidet, 2017).

\section{Méthodologie :}

Afin de confronter les aboutissements théoriques à la réalité marocaine, nous avons mené une étude qualitative auprès de 24 étudiants issus des établissements à accès régulé dans les domaines commerce, gestion et sciences de l'ingénieur. Des entretiens semi-directifs ont été réalisés afin de permettre aux étudiants de s'exprimer librement sur leurs perceptions du style d'enseignement et perception d'employabilité. La population de cette étude relève de spécialités et niveaux différents (commerce et gestion, sciences de l'ingénieur), provenant des établissements publics à accès limité comme (ENCG, ENSA, FSJES, FST), concernant la couverture géographique nous avons pu réaliser des entretiens avec des étudiants/diplômés des différents points géographiques (Rabat, Oujda, Tanger, Tetouan, Meknes, Fes, Agadir). Nous avons réalisé une analyse de contenu des réponses suivant la méthode d'analyse de contenu catégorielle et thématique. En effet, l'analyse par thème permet d'identifier par décomposition progressive du corpus de verbatims les idées phares développées (analyse sémantique) par les étudiants, considérés comme seule unité d'analyse de notre recherche.

Pour analyser et traiter les données collectées, Nous nous sommes basés sur le principe de saturation qui indique le seuil de la saturation théorique selon les redondances d'idées. C'est-à-dire que nous avons procédé au regroupement des verbatims qui portent la même signification, ou bien qui traitent la même idée. Conformément à la méthode d'analyse de contenu, Chaque groupe de verbatims triés a donné naissance ensuite à une dénomination. En d'autres termes, il a donné naissance à un thème.

Afin de construire les thèmes relatifs à cette étude, ainsi suivant la méthode de formulation des hypothèses de (Barth, 2007), l'hypothèse principale contient en germes les hypothèses de la recherche. Nous avons créé donc les thèmes relatifs à cette recherche en s'inspirant des aboutissements théoriques (hypothèses de la recherche) et des items proposés par les auteurs de référence. C'est ainsi que nous avons construit notre architecture thématique et notre guide d'entretien.

Les thèmes de l'étude sont les suivant : 1-La perception des étudiants marocains sur le style pédagogique instauré par le professeur.2l'autodétermination exprimée par les étudiants/Diplômés 3- Le sentiment d'efficacité personnelle 4- L'employabilité individuelle perçue. 
Après avoir présenté une analyse sémantique du corpus des verbatims, l'objectif est d'expliciter les croyances et les perceptions des étudiants afin d'étudier le lien et l'influence du style pédagogique autonomisant instauré par le professeur et la perception de l'employabilité chez les étudiants/diplômés. Dans ce qui va suivre nous allons présenter les résultats suivant la forme thématique de notre recherche.

\section{Analyse et discussions des résultats :}

\subsection{La perception des étudiants marocains sur le style pédagogique instauré par le professeur.}

L'objectif de cette section est de vérifier la perception des étudiants auprès du climat instauré par le professeur en classe ; autrement dit, nous avons tenté de collecter les informations pertinentes et les idées phares sur la perception des étudiants vis-à-vis le style pédagogique instauré par le professeur en classe (contrôlant, autonomisant...).

Tableau 1 : la perception des étudiants sur le style impersonnel instauré par le professeur

\begin{tabular}{|c|c|c|}
\hline Thème 1 & Fréquences d'idées & Exemples d'extraits de verbatim \\
\hline \multirow[t]{2}{*}{$\begin{array}{l}\text { Perceptio } \\
n \\
\text { d'autono } \\
\text { mie chez } \\
\text { les } \\
\text { étudiants / } \\
\text { Diplômés }\end{array}$} & $\begin{array}{l}\text { Un style } \\
\text { pédagogique } \\
\text { contrôlant est } \\
\text { identifié à raison de } \\
63 \% \text { de la population } \\
\text { interviewés }\end{array}$ & $\begin{array}{l}\text { - } \quad \text { il existe encore des professeurs qui adopte un } \\
\left.\text { style autoritaire en classe...» (CAS } N^{\circ} 10\right) \\
\text { - je ressens pas une autonomie en classe, car, à } \\
\text { mon sens, il n'y a pas cette volonté de } \\
\text { socialiser, comprendre et aider l'étudiant " } \\
\left.\text { CAS } N^{\circ} 3\right) \\
\text { "personnellement, je n'arrive pas à } \\
\text { m'exprimer ouvertement en classe, je ressens } \\
\text { une inquiétude quant à la réaction du } \\
\text { professeur et pour moi cela fait que cette } \\
\left.\text { relation est limitée »CAS } N^{\circ} 24\right)\end{array}$ \\
\hline & $\begin{array}{l}\text { Un style } \\
\text { pédagogique } \\
\text { autonomisant est } \\
\text { identifié à raison de } \\
37 \% \text { de la population } \\
\text { interviewés }\end{array}$ & $\begin{array}{l}\text { - } \quad \text { Oui, Le professeur nous accorde la liberté de } \\
\text { nous exprimer ouvertement sur des sujets } \\
\left.\text { différents » (CAS } N^{\circ} 15\right) \\
\text { - } \quad \text { En master, on a réussi à créer une bonne } \\
\text { ambiance de travail, c'est grâce à certains } \\
\left.\text { professeurs » (CAS } N^{\circ} 14\right) \\
\text { - Quand on se trouve avec des professeurs avec } \\
\text { qui on peut s'exprimer librement, ... la } \\
\text { discussion devient d'une grande importance } \\
\text { et d'une grande valeur ajoutée » }\end{array}$ \\
\hline
\end{tabular}

Dans le but d'identifier le style impersonnel instauré par le professeur en classe, plusieurs questions ont été adressées aux interviewés pour tenter de 
repérer le comportement des professeurs, la relation de confiance, l'encouragement en classe, s'exprimer ouvertement en classe etc.

Les résultats sont alarmants à ce niveau, en effet, un style autocratique et contrôlant en classe a été exprimé par la majorité des interviewés, et peu d'étudiant reconnaissent le climat autonomisant et motivant perçue en classe.

Ainsi, nous constatons par ailleurs, selon les résultats obtenus que le rôle des professeurs n'est pas limité au cadre du cours mais au-delà : « ... le professeur à mon avis joue un rôle important même au niveau du changement des croyances, principes, personnalité de l'étudiant. »(CAS $\left.N^{\circ} 12\right)$, "Quand on se trouve avec des professeurs avec qui on peut s'exprimer librement, la discussion avec ces professeurs en cours ou en dehors du cours devient d'une grande importance et d'une grande valeur ajoutée » (CAS N ${ }^{\circ}$ )

Ceci explique l'influence du professeur sur l'identité des étudiants, et cela, selon notre analyse ne serait possible sans créer un climat autonomisant.

\subsection{L'autodétermination exprimée par les étudiants/Diplômés}

Concernant l'autodétermination des étudiants, selon les résultats, il semble que la tendance des répondants est en faveur d'une motivation extrinsèque, alors que la motivation intrinsèque semble seulement en faveur de 5 interviewés sur 24.

Les raisons qui poussent les étudiants/diplômés à travailler de manière extrinsèque sont différentes, toutefois, les idées exprimées par les interviewés font souvent référence à la «note», qui reste l'élément central forçant l'étudiant à travailler : "...je serai sanctionné par la note si je ne fais pas mes

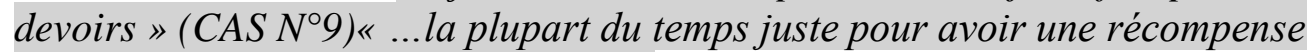
de la part du professeur » $\left(C A S N^{\circ} 19\right) \ldots$

On peut citer également la pression psychologique des professeurs qui peut influencer les étudiants :

«Je fais mes devoirs et mes recherches juste pour pouvoir passer mes examens » (CAS $\left.N^{\circ} 3\right)$ «... Il y'a même des fois ou le professeur vous force à faire quelque chose ... » (CAS $\left.N^{\circ} 5\right)$...

Parfois, il semble carrément que les étudiants ressentent un sentiment de culpabilité de ne pas participé en classe,

"Parfois, je participe en classe pour ne pas m'ennuyer, parfois on n'a pas le choix » $\left(C A S N^{\circ} 7\right)$, "Je veux que le professeur pense que je suis une bonne étudiante » $\left(C A S N^{\circ} 11\right) \ldots$ 
Tableau 2 : l'autodétermination exprimée par les étudiants/Diplômés

\begin{tabular}{|c|c|c|}
\hline Thème 2 & Fréquences d'idées & Exemples d'extraits de verbatim \\
\hline \multirow[t]{2}{*}{$\begin{array}{l}\text { L'autodétermin } \\
\text { ation des } \\
\text { étudiants }\end{array}$} & $\begin{array}{l}5 \text { interviewés sur } 24 \\
\text { s'identifient à une } \\
\text { motivation intrinsèque, }\end{array}$ & $\begin{array}{l}\text { - } \quad \text { "La raison c'est premièrement l'amour } \\
\left.\text { à la matière » (CAS } N^{\circ} 19\right) \\
\text { - } \quad \text { Je le fais par motivation et par amour } \\
\left.\text { pour atteindre mon objectif (cas } N^{\circ} 4\right) \\
\text { - J'ai tendance à investir mon temps pour } \\
\text { l'intérêt et le plaisir que je porte pour la } \\
\text { matière (cas N2) } \\
\text { - } \quad \text { "Je fais mes devoirs par intérêt et plaisir, } \\
\text { surtout quand j'apprécie le professeur et } \\
\text { la matière »(Cas N22) }\end{array}$ \\
\hline & $\begin{array}{l}19 \text { interviewés sur } 24 \\
\text { déclarent une motivation } \\
\text { extrinsèque }\end{array}$ & $\begin{array}{l}\text { - "...la plupart du temps juste pour avoir } \\
\text { une bonne impression de la part du } \\
\left.\text { professeur »(CAS } N^{\circ} 9\right) \\
\text { - } \\
\text { - "Je travaille pour obtenir une bonne } \\
\left.\text { note à la fin de l'année... »(Cas } N^{\circ} 8\right) \\
\text { - «... Il y'a même des fois où le professeur } \\
\text { vous force à faire quelque chose ... » } \\
\left.\text { (CAS } N^{\circ} 5\right) \\
\text { «Parfois, je participe en classe pour ne } \\
\text { pas m'ennuyer, parfois on évite la } \\
\text { sanction du professeur }\left(C A S N^{\circ} 7\right)\end{array}$ \\
\hline
\end{tabular}

Afin d'étudier le thème relatif à l'autodétermination nous nous sommes inspirés des travails liés aux contexte éducatif. Ainsi, d'autre part, pour évaluer son lien avec l'employabilité, Nous avons privilégié les items de G. Camus et al (2017) qui ont proposés indicateurs de mesure permettant de mesurer la motivation à poursuivre l'objectif futur de (re)travailler vu par la Théorie de L'autodétermination. Dans ce sens, deux questions ont été retenu :

- Vous imaginez que votre travail futur sera une source d'épanouissement et de plaisir pour vous ?

- Quelle est la situation qui vous correspond le mieux : - j'aime Travailler, c'est intéressant, et ça permettra de se sentir mieux dans d'autres domaines - travailler c'est gagner plus d'argent et me sentir mieux financièrement- travailler c'est pour éviter d'être de critiquer par les proches ou autre chose à proposer ?

Les résultats de notre étude montrent une orientation générale vers la motivation extrinsèque, pratiquement la majorité des interviewés souhaite dans le futur un travail en vue d'améliorer en premier lieu leur situation financière. Cependant, l'importance n'est pas vitale si le travail futur représente une source d'épanouissement. Nous retenons donc, 15/24 
personnes cherchant en premier lieu une source financière considérée comme un besoin physiologique nécessaire : "en premier lieu un travail sécurisant pour moi sera une source d'argent »cas $N^{\circ} 9$, «je ne peux pas considérer le travail comme source d'épanouissement sans être d'abord d'être satisfaite financièrement » cas $N^{\circ} 6$, "le premier objectif pour moi c'est gagner de l'argent $\gg$ cas $N^{\circ} 10$.

Pour le reste, $6 / 24$ s'identifiant à une motivation intrinsèque, car les interviewés dans ce cas souhaitent en premier lieu un travail futur épanouissant : "le travail pour moi sera une grande source de stress et dépression si j'aime pas mon travail»(Cas $\left.N^{\circ} 2\right)$, " je pense que le travail et gagner de l'argent sont corrélés, travailler par amour et intérêt $=$ travailler bien = plus d'argent $»\left(\right.$ Cas $\left.N^{\circ} 19\right)$, " pour moi le travail sera d'abord une source de plaisir et d'épanouissement $»\left(\right.$ Cas $\left.N^{\circ} 4\right)$

Nous constatons que pratiquement les mêmes personnes qui se sont identifiées à une motivation extrinsèque en premier lieu dans un contexte académique d'apprentissage sont relativement les mêmes identifiées à une motivation extrinsèque lorsqu'il sont interrogés sur le travail futur, ce qui peut expliquer, l'idée que la motivation autodéterminée résultante d'un style pédagogique autonomisant conduit à réguler le comportement et la perception des étudiants/diplômés vers l'autodétermination en contexte académique et probablement vers d'autres contextes et le contraire est vrai.

\subsection{Le sentiment d'efficacité personnel}

Les résultats de notre étude confirment l'importance du style impersonnel instauré par le professeur et son effet sur le sentiment d'efficacité personnel des étudiants/diplômés, autrement dit, en contexte universitaire, le sentiment d'efficacité ne serait élevé que si et seulement si, l'étudiant/diplômé s'aperçoit dans un climat en classe autonomisant et motivant et l'activité à réaliser est jugé intéressante pour l'étudiant.

L'objectif étant de vérifier les croyances d'efficacité dans un contexte académique caractérisé par un style autonomisant et motivant et d'estimer le niveau de croyances d'efficacité face à des situations diverses :

Les idées exprimées par les interviewés indiquent une légère tendance vers le manque de croyances d'efficacité des étudiants/diplômés. Comme le montre le tableau ci-dessous : 
Tableau 3 : Tableau de fréquences d'idées sur les croyances d'éfficacité exprimées par les étudiants/Diplômés

\begin{tabular}{|c|c|c|}
\hline Thème & Fréquences d'idées & Extraits de verbatim \\
\hline \multirow{2}{*}{$\begin{array}{l}\text { Le } \\
\text { sentiment } \\
\text { d'efficacité } \\
\text { chez les } \\
\text { étudiants }\end{array}$} & $\begin{array}{l}83 \% \text { de la population } \\
\text { identifiée à un sentiment } \\
\text { d'efficacité élevée dans } \\
\text { une situation de vie }\end{array}$ & $\begin{array}{l}3 \text { «Je persiste pour arriver à la solution de } \\
\left.\text { l'exercice. »(CAS } N^{\circ} 9\right) \\
4 \quad \text { "Quand je fixe un objectif je dois le réaliser dans } \\
\left.\text { n'importe quelle situation » (CAS } N^{\circ} 2\right) \\
5 \text { "J'essaie de trouver d'autre manières pour le } \\
\text { comprendre, si je me bloque j'essaie d'autres } \\
\text { solutions, je persiste à n'importe quelle situation" } \\
\left(C A S N^{\circ} 1\right)\end{array}$ \\
\hline & $\begin{array}{l}54 \% \text { identifié à un } \\
\text { sentiment d'efficacité } \\
\text { relativement faible dans } \\
\text { la situation réelle vécu } \\
\text { par les étudiants }\end{array}$ & $\begin{array}{l}6 \text { "Mon effort va dépendre de l'activité à réaliser, } \\
\text { et aussi du professeur» }\left(C A S N^{\circ} 14\right) \\
7 \text { "si c'est un travail stressant, et pas intéressant, je } \\
\left.\text { peux abandonner facilement»(CAS } N^{\circ} 21\right) \\
8 \quad \text { "... il y a des étudiants brillants et compétents plus } \\
\left.\text { que moi» (CAS } N^{\circ} 19\right) \\
9 \quad \text { "...mes capacités d'études, Pas excellente mais je } \\
\left.\text { dirai moyenne ... »(CAS } N^{\circ} 7\right) \\
10 \quad \text { "Plus ou moins, parfois je sens que mes capacités } \\
\text { d'études sont excellentes parfois non» (CAS } \\
\left.N^{\circ} 16\right)\end{array}$ \\
\hline
\end{tabular}

Les résultats de notre étude explique le rôle important du professeur dans le renforcement des croyances d'efficacité des étudiants/diplômés : « Mon effort va dépendre de l'activité à réaliser, et aussi du professeur » (CAS $\left.N^{\circ} 14\right)$ « En classe, je pense que le professeur peut guider et influencer l'étudiant à réaliser une activité »(Cas 23), il semble donc évident, qu'un professeur par la persuasion verbale influence positivement sur la motivation et l'intérêt à réaliser une activité. D'autres part, les résultats indiquent, que si l'étudiant se sent stressé ou troublé quant à la réalisation de l'activité, il est fort probable que l'étudiant/diplômé se désengage rapidement de la réalisation de l'activité, surtout s'il s'agit d'une tâche à caractère non obligatoire. Par contre, dans le cas où l'étudiant se sent confiant et alaise vis-à-vis cette activité, il est fort probable que l'étudiant s'investit beaucoup pour la réaliser : " si c'est un travail stressant, et pas intéressant, je peux abandonner facilement»(CAS $\left.N^{\circ} 21\right)$

, "Par contre si j'ai affaire à quelque chose que je n'aime pas, je fais le minimum d'effort possible, et si ce n'est pas obligatoire de le faire, je peux même abandonner » $\left(C A S N^{\circ} 3\right)$ 
Compte tenu de l'aspect médiateur de cette variable, dans le sens où elle se pose comme résultat d'influence du soutien à l'autonomie du professeur, et explicatif pour la variable de l'employabilité.

Nous nous sommes inspirés de Pintrich (1991)《 Motivated Strategies for Learning Questionnaire » qui permet une adaptation des items à toutes les situations.et donc le premier volet du tableau numéro3 ( tableau ci-dessous) permet de décrire le sentiment d'efficacité face à des situations multiples. Dans ce sens, l'auto-efficacité des étudiants est généralement considéré élevée; selon les entretiens réalisés, $80 \%$ de la population se croit capable d'affronter les problèmes qui surviennent dans la vie, et se croit capable de réaliser toutes les activités ( travail, vie), et généralement ont confiance en leurs capacités ...

"Quand je fixe un objectif je dois le réaliser dans n'importe quelle situation » $\left(C A S N^{\circ} 2\right)$ «'essaie toujours de trouver une solution pour faire le travail, soit en demandant de l'aide ou je trouve une autre manière pour faire le travail » $\left(C A S N^{\circ} 6\right)$ « Je fais partie des gens qui persiste coute que coute » $\left(C A S N^{\circ} 4\right)$

Nous pouvons retenir donc que le climat en classe permet d'expliquer la hausse ou la baisse du niveau d'auto-efficacité sans pour autant dire que le niveau d'auto-efficacité explique le développement de l'employabilité, il semble donc plus judicieux de développer ou trouver un outil de mesure plus déterminant et plus solide qui permet de présenter une explication plus convaincante sur la relation du sentiment d'efficacité personnel sur l'employabilité

\section{4. l'employabilité individuelle perçue}

L'employabilité reste le centre de préoccupation de notre recherche, nous tenons à rappeler que les questions qui ont été adressés aux jeunes étudiants / diplômés se concentrent sur la dimension « étudiant», et se consacre à la recherche de développement des caractéristiques individuelles. L'ensemble des sous thèmes retenus sont: communication, performance académique, intelligence émotionnelle, expériences vie/travail, orientation entrepreneuriale. 
Tableau 4: Tableau de fréquences d'idées sur le thème de l'employabilité des diplômés dans une approche basée sur les compétences

\begin{tabular}{|c|c|c|c|}
\hline Thème & Sous-thèmes & Fréquences d'idées & Extraits de verbatim \\
\hline \multirow[t]{3}{*}{$\begin{array}{l}\text { Employabil } \\
\text { ité des } \\
\text { diplômés }\end{array}$} & $\begin{array}{l}\text { Academic } \\
\text { performance \& } \\
\text { study skills }\end{array}$ & $\begin{array}{l}95 \% \text { de la population } \\
\text { interviewée } \\
\text { considèrent que le } \\
\text { dispositif formation } \\
\text { au Maroc n'estpas en } \\
\text { adéquation vis-à-vis } \\
\text { les attentes des } \\
\text { étudiants. }\end{array}$ & $\begin{array}{l}\text { "Je ne suis pas très satisfait, car, ce qu'on a } \\
\text { acquis n'est pas suffisant pour trouver un } \\
\text { emploi aujourd'hui... »(CAS } N^{\circ} 11 \\
\text { "je ne suis pas satisfait, car, les compétences et } \\
\text { connaissances que nous avons acquis sont } \\
\left.\text { dépassées. » (CAS } N^{\circ} 1\right) \\
\text { "Non je ne suis pas suffisamment satisfaite, ce } \\
\text { qu'on a appris est totalement différent de la } \\
\left.\text { réalité » (CAS } N^{\circ} 3\right) \text {.... } \\
\text { "Le monde est en train de changer alors que le } \\
\text { système académique ne change pas ... » (CAS } \\
\left.N^{\circ} 17\right)\end{array}$ \\
\hline & Generic skills & $\begin{array}{l}75 \% \text { des interviewés } \\
\text { expriment le manque } \\
\text { d'importance } \\
\text { accordées aux } \\
\text { compétences } \\
\text { transversales }\end{array}$ & $\begin{array}{l}\text { "personnellement, je pense que les } \\
\text { compétences acquises au cours de mon } \\
\text { parcours sont très techniques }\left(C A S N^{\circ} 6\right) \\
\text { "la formation que j'ai acquise se concentre sur } \\
\text { le volet technique et laisse de côté des aspects } \\
\text { important comme le développement de la } \\
\text { personnalité de l'étudiant (cas } N^{\circ} 19 » \\
\text { "Je pense que notre domaine nécessite aussi } \\
\text { des compétences non techniques importantes } \\
\text { qui ont été, a mon sens, souvent } \\
\text { marginalisées }\left(C A S N^{\circ} 12\right) »\end{array}$ \\
\hline & Communication & $\begin{array}{l}70 \% \text { de la population } \\
\text { interviewée trouvent } \\
\text { des difficultés au } \\
\text { niveau } \\
\text { communication }\end{array}$ & $\begin{array}{l}\text { "On n'accorde pas une grande importance à la } \\
\text { communication dans notre spécialité, ce qui } \\
\left.\text { compte c'est le technique »(CAS } N^{\circ} 9\right) \\
\text { " je trouve des difficultés au niveau des } \\
\text { exposés » }\left(C A S N^{\circ} 24\right) \\
\text { "on est en } 5 \text {-ème année et vraiment on n'a pas } \\
\text { l'audace de parler en public,» }\left(C A S N^{\circ} 10\right)\end{array}$ \\
\hline
\end{tabular}

L'employabilité à été traitée sur deux niveaux différents :

Dans une approche de l'employabilité basé sur les compétences, au niveau des compétences basiques, qui constituent le rendement de l'université/école marocaine; Les résultats de notre étude montrent une insatisfaction générale sur le système de formation marocain, puisque, $95 \%$ de la population interviewée considère que le dispositif formation au Maroc n'est 
pas en adéquation avec les attentes de l'employeur, et ne permet pas de suivre l'évolution socio-économique.

Ensuite, nous avons tenté de vérifier la pertinence d'intégrer des compétences non techniques, dites transversales, inspiré du modèle de D.Pool et AL (2007),

Les compétences sociales et entrepreneuriales sont considérées comme l'élément phare de cette section, car, pour la plupart des étudiants/diplômés, en fait, 17 sur 24 personnes ont exprimés des problèmes de communication et 21 personnes sur 24 se déclare incapable de créer ou gérer un business, soit donc un manque d'orientation entrepreneurial.

La seconde approche de l'employabilité est basée sur des qualités individuelles considérées au niveau de la revue de littérature comme la clé de développement de l'employabilité des jeunes diplômés :

Tableau 5: Fréquences d'idées sur le thème de l'employabilité des diplômés dans une approche basée sur les qualités individuelles

\begin{tabular}{|c|c|c|c|}
\hline Thème & Sous thème & $\begin{array}{l}\text { Fréquences } \\
\text { d'idées }\end{array}$ & Extrait du verbatim \\
\hline \multirow[t]{3}{*}{$\begin{array}{l}\text { Employabilité } \\
\text { des diplômés }\end{array}$} & $\begin{array}{l}\text { Orientation } \\
\text { entrepreneuriale }\end{array}$ & $\begin{array}{l}87 \% \text { expriment } \\
\text { l'incapacité de } \\
\text { gérer ou créer } \\
\text { un business }\end{array}$ & $\begin{array}{l}11 \text { «A mon avis, ce n'est pas important, je } \\
\text { ne suis pas vraiment intéressé à ça " } \\
\left.\text { (CAS } N^{\circ} 7\right) \\
12 \text { "On avait une matière qui porte sur } \\
\text { l'entreprenariat, ... si je crée mon } \\
\text { entreprise, j'aurais certainement } \\
\text { besoin de l'aide sur ce niveau » (CAS } \\
N^{\circ} 9 \text { ) } \\
13 \text { Je pense que mes compétences sont } \\
\text { insuffisantes .... On n'a pas la capacité } \\
\text { de prendre la responsabilité, de gérer, } \\
\text { de prendre des décisions... » (CAS } \\
\left.N^{\circ} 14\right)\end{array}$ \\
\hline & $\begin{array}{l}\text { Intelligence } \\
\text { émotionnelle }\end{array}$ & $\begin{array}{l}16 / 24 \text { trouvent } \\
\text { des difficultés } \\
\text { sur le contrôle } \\
\text { et gestion des } \\
\text { émotions }\end{array}$ & 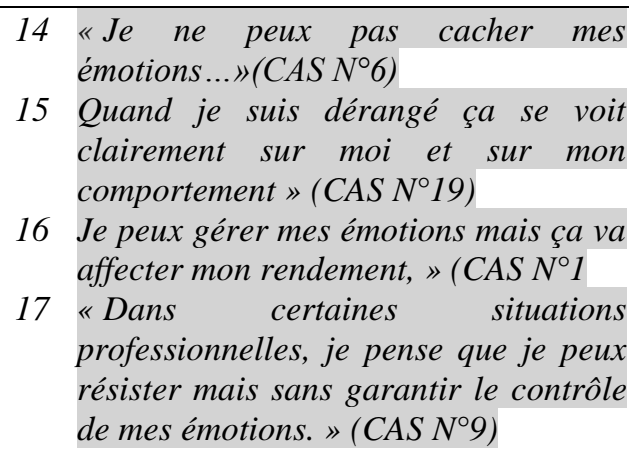 \\
\hline & & $\begin{array}{l}17 / 24 \\
\text { Déclarent }\end{array}$ & $\begin{array}{l}18 \text { "Oui j'ai fait des stages, mais la vérité } \\
\text { je n'ai pas appris beaucoup de } \\
\left.\text { choses...»(CAS } N^{\circ} 3\right)\end{array}$ \\
\hline
\end{tabular}




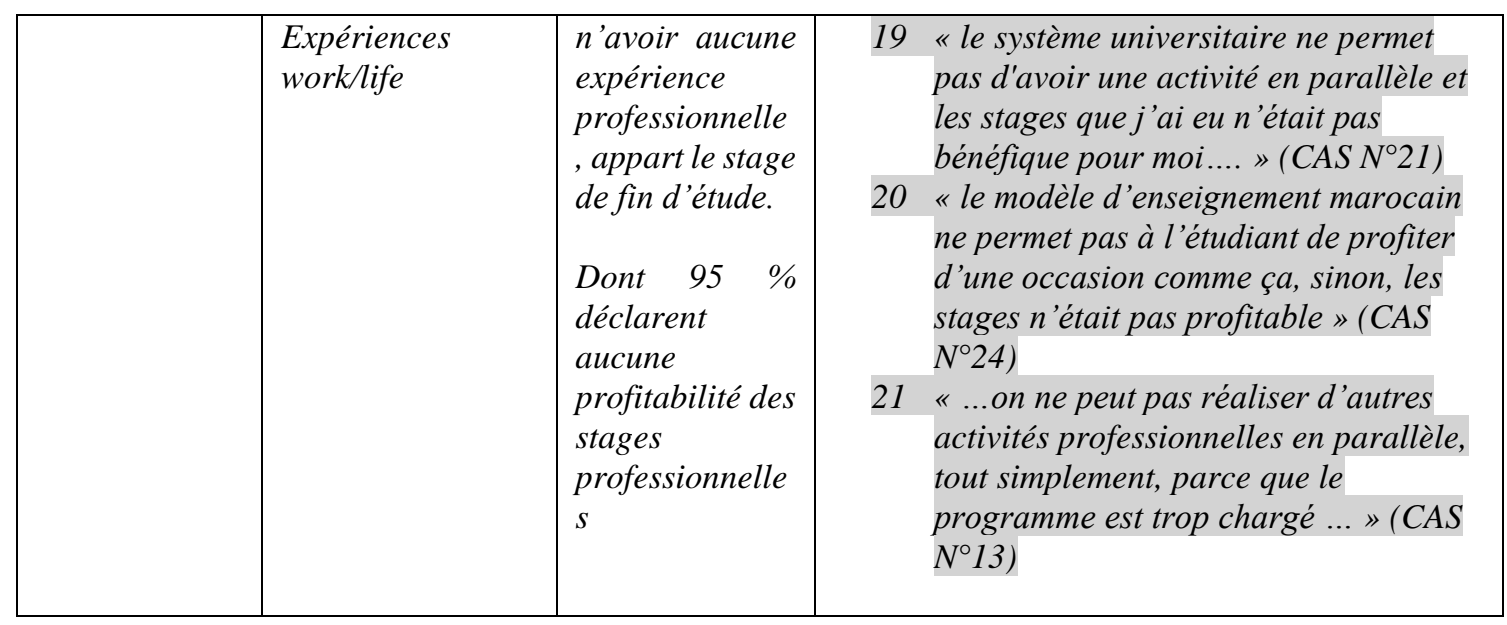

L'étude fait des constats importants sur l'intelligence émotionnelle des étudiants, en effet, les jeunes étudiants/diplômés, à raison de $66 \%$ des interviewés déclarent un manque de contrôle et gestion des émotions, par ailleurs les $44 \%$ restants, estiment avoir une capacité de gérer leurs états émotionnels ou dans les meilleurs des cas l'état émotionnel de leurs entourages. Toutefois, pratiquement tous les interviewés estiment que l'intelligence émotionnelle peut s'apprendre à l'université.

Deuxièmement, notre modèle met en évidence l'importance d'avoir des qualités personnelles provenant d'une expérience de vie/travail, $71 \%$ des répondants ont soulignés l'aspect non profitable de l'expérience de stage, à ce niveau la situation semble alarmante.

Seulement $16 \%$ de la population de l'étude, soit 4 sur 24 ont pu réaliser, en plus des périodes de stages, des activités professionnelles ou associatives, en parallèle avec leurs parcours d'études. Il parait que cette catégorie est la plus satisfaite de l'expérience professionnelle, en effet tous les répondants ont déclaré l'existence d'un grand écart entre l'expérience professionnelle de travail dans le cadre d'un contrat de travail et l'expérience dans le cadre d'un stage.

Suivant l'analyse de notre corpus thématique, il semble qu'effectivement les professeurs qui développent un style émancipateur développent chez les étudiants des croyances sur un sentiment de confiance et d'autodétermination et d'auto efficacité car les réponses semblent en corrélation sur les différents thèmes présentés dans le cadre de notre étude. Il parait qu'un style émancipateur créent ou du moins améliore chez les étudiants des croyances quant à leurs capacité de se développer mais aussi de trouver les ingrédients nécessaires à leurs employabilité alors que les étudiants qui perçoivent un style contrôlant auto rapporté par l'enseignant trouvent des difficultés et ressentent particulièrement un manque quant à leurs croyances et 
leurs confiance vis-à-vis le développement des compétences et des caractéristiques individuelles nécessaires à leurs carrière futurs. Il en suit que l'autodétermination et l'auto efficacité représentent effectivement un pont quant à la perception des étudiants de leurs employabilité. Dans le sens où, les étudiants qui développent des croyances d'efficacité élevée et une autodétermination trouvent la motivation, la confiance et les qualités individuelles qui déterminent leurs épanouissements personnels. Nous pouvons donc avancer qu'un style pédagogique autonomisant qui favorise les trois besoins psychologiques contribue au développement identitaire de l'étudiant et développent des perceptions chez les étudiants quant à leurs niveaux d'employabilité.

Le soi représente donc un élément essentiel à la malléabilité de l'état d'esprit de l'étudiant, dans le sens où, l'employabilité peut s'influencer par les perceptions individuelles. En effet, cette malléabilité fait qu'un étudiant soit plus susceptible de percevoir un problème comme une opportunité d'apprentissage, voire une occasion de démontrer la compétence. A partir de cette idée, nous pouvons déduire que le soi joue un rôle important au niveau de la perception des étudiants quant à leurs niveau d'employabilité, et par conséquent, la perception de l'employabilité est un indicateur de l'employabilité réelle.

\section{Conclusion}

Aujourd'hui, l'analyse de l'employabilité des diplômés est la question centrale qui devient de plus en plus prioritaire dans le contexte éducatif marocain, la présente étude à démontrer plusieurs aspects importants de l'employabilité. Dans ce sens, nous constatons que plusieurs travaux théoriques et empiriques se concentrent à analyser ce concept du point de vue économique et managériale, dans le cas de notre étude, nous avons proposé une vision sociologique et psychologique de l'employabilité qui se concentre sur les compétences et les qualités individuelles des étudiants.

Ce travail, focalise sur l'étudiant comme unique unité d'analyse, il parait que la majorité des travaux francophones se concentre sur la vision de l'employeur afin de répondre à la problématique de l'employabilité. Dans ce travail, nous avons montré qu'il est effectivement important d'ajouter une nouvelle dimension à l'analyse de l'employabilité basée sur les croyances et les perceptions des étudiants.

Les résultats obtenus confirment la validité de notre modèle théorique, ainsi que les dimensions de l'employabilité " analysé du point de vue pédagogique » trouvent leurs importances dans le contexte marocain. Il en découle que la clé pour améliorer l'employabilité des jeunes marocains, selon cette étude, réside donc le développement des caractéristiques individuelles et des compétences surtout non techniques comme les compétences sociales et 
entrepreneuriales. Qui sont aujourd'hui très sollicitées dans la vie socioéconomique.

Effectivement, la pédagogie universitaire joue un rôle fondamental dans le développement d'une pré-employabilité des étudiants, l'émancipation et l'épanouissement des étudiants représente comme le démontre ce travail, une source importante au développement d'une employabilité réelle. Finalement la confirmation et la généralisation de ces aboutissements nécessite une étude confirmatoire quantitative qui permettra éventuellement de toucher un grand échantillon et de conclure avec des leviers et préconisations sur les pratiques et méthodes pédagogiques susceptible d'impacter les caractéristiques individuelles des étudiants.

\section{References:}

1. Altet, M. (1994). Comment interagissent enseignant et élève en classe : Note de synthèse. Revue française de pédagogie, 107(1), 123-139. https://doi.org/10.3406/rfp.1994.1268

2. Andreani, J.-C. (2005). Méthodes d'analyse et d'interprétation des études qualitatives : État de l'art en marketing. scienceopen, 26.

3. Bandura, A. (2003). Auto-efficacité : Le sentiment d'efficacité personnelle, (2ème édition). De Boeck.

4. Black, A., \& Deci, E. (2000). The Effects of Instructors' Autonomy Support and Students' Autonomous Motivation on Learning Organic Chemistry: A Self-Determination Theory Perspective. John Wiley \& Sons, 17.

5. Camus, G., Berjot, S., Amoura, C., \& Forest, J. (2017). Échelle de motivation à (re)travailler: Vers une nouvelle approche de la theórie de l'autodétermination. Canadian Journal of Behavioural Science, 49. https://doi.org/10.1037/cbs0000072

6. Carré, P. (2004). Bandura: Une psychologie pour le XXIe siècle ? Savoirs, Hors série(5), 9. https://doi.org/10.3917/savo.hs01.0009

7. Dacre Pool, L., Qualter, P., \& J. Sewell, P. (2014). Exploring the factor structure of the CareerEDGE employability development profile. Education + Training, 56(4), 303-313. https://doi.org/10.1108/ET-012013-0009

8. Dacre Pool, L., \& Sewell, P. (2007). The key to employability: Developing a practical model of graduate employability. Education + Training, 49(4), 277-289. https://doi.org/10.1108/00400910710754435

9. Deci, E. L., \& Ryan, R. M. (1985). The general causality orientations scale: Self-determination in personality. Journal of Research in Personality, 19(2), 109-134. https://doi.org/10.1016/00926566(85)90023-6 
10. Frimousse, S., Hofaidhllaoui, M., Swalhi, A., \& Zgoulli, S. (2013). L'épuisement des cadres au sein des PME: le rôle de l'employabilité. AGRH. 19.

11. Fugate, M., \& Kinicki, A. J. (2008). A dispositional approach to employability : Development of a measure and test of implications for employee reactions to organizational change. Journal of Occupational and Organizational Psychology, 81(3), 503-527.

https://doi.org/10.1348/096317907X241579

12. Fugate, M., Kinicki, A. J., \& Ashforth, B. E. (2004). Employability: A psycho-social construct, its dimensions, and applications. Journal of Vocational Behavior, 65(1), 14-38. https://doi.org/10.1016/j.jvb.2003.10.005

13. Harvey, L. (2001). Defining and Measuring Employability. Quality in Higher Education, 7(2), 97-109. https://doi.org/10.1080/13538320120059990

14. Hofaidhllaoui, M., \& Vatteville, E. (2011). Mesurer l'employabilité des jeunes diplômés: L'exemple des titulaires de masters tunisiens. Colloque AGRH, 19.

15. Hofaidhllaoui, M \& Roger, M. (2014) « Favoriser le développement de l'employabilité : un enjeu pour les individus et pour les organisations », Revue de gestion des ressources humaines, 2014/3 ${ }^{\circ}$ 93, p. 32-53. DOI : 10.3917/grhu.093.0032

16. Jamel, O. (2011). Jamel Othmane, L'employabilité : Définition, création d'une échelle de mesure et contribution à l'étude des déterminants | isidore.science [université moulin III de lyon]. https://isidore.science/document/10670/1.yc6oaw

17. Knight, P. (2004). Learning, Curriculum and Employability in Higher Education (1 $\quad$ éd. $)$ Routledge. https://doi.org/10.4324/9780203465271

18. Knight, P., \& Yorke, M. (2004). Learning, Curriculum and Employability in Higher Education. Psychology Press.

19. Leroy, N., Bressoux, P., Sarrazin, P., \& Trouilloud, D. (2013). Un modèle sociocognitif des apprentissages scolaires: Style motivationnel de l'enseignant, soutien perçu des élèves et processus motivationnels. Revue française de pédagogie. Recherches en éducation, 182, 71-92. https://doi.org/10.4000/rfp.4008

20. Mantze, Y., \& Knight, P. (2006). Embedding employability into the curriculum Yorke and Knight.pdf. learning and employability series 1.

21. Reeve, J. (2006). Teachers as Facilitators: What AutonomySupportive Teachers Do and Why Their Students Benefit. The Elementary School Journal, 106(3), 225-236. https://doi.org/10.1086/501484 
22. Reeve, J., Ryan, R., \& Deci, E. (s. d.). Self determination theory: A dialectical framework for understunding the socio-cultural influences on student motivation. CT:Information Age press, 4.

23. Rondier, M. (2004). A. Bandura. Auto-efficacité. Le sentiment d'efficacité personnelle. L'orientation scolaire et professionnelle, 3.

24. Said, B., \& Issam, M. (2020). Le style pédagogogique autonomisant contribue-t-il au développement d'une pré-employabilité des jeunes diplômés : Revue de littérature et proposition d'un modèle conceptuel. European Scientific Journal ESJ, 16(4). https://doi.org/10.19044/esj.2020.v16n4p107

25. Saint-Germes, È. (2004).L'employabilité, une nouvelle dimension de la grh, 15ème congrès de l'AGRH Montréal, sept. 2004 Communication primée : Bourse d'excellence de la Chaire en Gestion des Compétences de l'UQAM (Université du Québec à Montréal).

26. Sarrazin, P., Luc, P., Deci, E., \& Ryan, R. (2011). Chapitre 13 : Nourrir une motivation autonome et des conséquences positives dans différents milieux de vie : Les apports de la théorie de l'autodétermination (1re édition).

De

Boeck. https://www.researchgate.net/publication/331297361_Nourrir_une_m otivation_autonome_et_des_consequences_positives_dans_differents _milieux_de_vie_les_apports_de_la_theorie_de_l'autodetermination

27. Sarrazin, P., Tessier, D., \& Trouilloud, D. (2006). Climat motivationnel instauré par l'enseignant et implication des élèves en classe: L'état des recherches. Revue française de pédagogie, 157, 147-177. https://doi.org/10.4000/rfp.463

28. Thierry, côme, \& Abdellilah, Y. (2015). Accès régulé à l'Université et implication et motivation des étudiants: L'exemple du Maroc. Gestion et management public, Volume 3(4), 5-26. 